\title{
Síndrome de disfunción cognitiva de perros geriátricos
}

\section{Cognitive dysfunction syndrome in senior canines}

\author{
Diana Gallego V, ${ }^{1}$ MV, Judith Figueroa R, ${ }^{1}$ M.Sc, Camilo Orozco S, ${ }^{1 *}$ Ph.D. \\ ${ }^{1}$ Universidad Nacional de Colombia, Facultad de Medicina Veterinaria y de Zootecnia, Departamento \\ de Ciencias para la Salud Animal, Sede Bogotá, Colombia.*Correspondencia: caorozcos@unal.edu.co
}

Recibido: Noviembre 15 de 2009; Aceptado: Julio 18 de 2010.

\section{RESUMEN}

Durante su vida geriátrica, algunos perros pueden presentar cambios progresivos en su comportamiento habitual, como desorientación, disminución en la interacción con los miembros de la familia, irritabilidad y vocalización excesiva, entre otros. La inespecificidad de estas alteraciones ha hecho que muchas de estas modificaciones comportamentales sean consideradas, erróneamente, como signos típicos del envejecimiento "normal". No obstante, estas pueden ser reflejo de un proceso patológico cerebral que provoca deterioro progresivo de las funciones cognitivas en perros geriátricos. Recientemente, fue descrita una enfermedad conocida como Síndrome de Disfunción Cognitiva de perros senior o "enfermedad de Alzheimer del perro", debido a las similitudes clínicas y patológicas con esta enfermedad en humanos. Los animales afectados desarrollan cambios morfofuncionales en diferentes zonas cerebrales, lo que probablemente conduce a la aparición de cambios asociados al decline cognitivo, es decir, disminución en la capacidad de recopilar información, procesarla, retenerla y tomar decisiones, lo que termina provocando efectos deletéreos sobre la calidad de vida del paciente afectado al no lograr desenvolverse normalmente en su medio. Dada la inespecificidad de los signos clínicos, la anamnesis y los test comportamentales han sido considerados como las principales herramientas diagnósticas para identificar este síndrome. Por otra parte, debido a que aún no han sido plenamente establecidos los aspectos etiopatológicos, los mecanismos implicados en el desarrollo de esta neuropatología aún siguen inconclusos, por lo tanto, no son muchas las estrategias terapéuticas disponibles, de las cuales solo la selegilina ha sido aprobada por la FDA.

Palabras clave: Disfunción cognitiva canina, envejecimiento canino, neurodegeneración, síndrome demencial canino. 


\section{ABSTRACT}

During their senior life, some dogs may show progressive changes in their normal behavior such as disorientation, decreased interaction with family members, irritability and excessive vocalization, among others. The non-specificity of these alterations has led to the erroneous belief that many of these behavioral modifications are, typical or "normal" signs of aging. However, this may be a reflection of a pathological process that causes progressive brain deterioration of the cognitive functions in senior dogs. Recently, a condition called Cognitive Dysfunction Syndrome in senior dogs or "dog Alzheimer's disease" was described, due to its clinical and pathological similarities with the human disease. Affected animals develop morphological and functional changes in different areas of the brain, which probably leads to the appearance of changes associated with cognitive decline, i.e., decreased ability to gather, process, or retain information, and make decisions, which ends up causing deleterious effects on quality of life of affected patients by failing to function normally within their environment. Given the nonspecific clinical signs, medical history and behavioral tests have been considered as the main diagnostic tools to identify this syndrome. On the other hand, due to the fact that no etiopathological aspects have been fully established, the mechanisms used in the development of this neuropathology are still inconclusive, therefore, they are not many therapeutic strategies available, out of which Selegiline has been approved by the FDA.

Key words: Canine cognitive dysfunction, canine aging, neurodegeneration, canine dementia syndrome.

\section{INTRODUCCIÓN}

Desde tiempos ancestrales, el perro ha acompañado al hombre a lo largo de su historia evolutiva brindándole compañía, lealtad y seguridad. Dada esta estrecha relación, actualmente el canino es considerado como una de las especies domésticas más cercana al hombre, desempeñando un papel relevante como miembro activo en un gran número de familias y comunidades humanas alrededor del mundo donde, el sentido de responsabilidad de algunos propietarios por sus mascotas caninas, ha promovido el mejoramiento de los cuidados nutricionales y sanitarios que permiten al animal, alcanzar, alargar y vivir normalmente su vejez (1). No obstante, la edad avanzada en caninos se asocia frecuentemente a alteraciones comportamentales y déficit cognitivo, los cuales pueden manifestarse con signos clínicos de desorientación, pérdida de la interacción social, disturbios del sueño, disminución en la actividad general y pérdida progresiva de recuerdos adquiridos durante el entrenamiento casero o profesional (1-3). Inicialmente, estos cambios comportamentales fueron asumidos por algunos médicos veterinarios, como indicio de senilidad asociada a la edad, de etiología desconocida y frente a los cuales no se podía realizar ningún tipo de intervención (2). Sin embargo propusieron la terminología de Síndrome de Disfunción Cognitiva de perros geriátricos (SDC), para describir el déficit cognitivo observado en algunos perros geriátricos $(1,2,4,5)$.

EI SDC o "enfermedad de Alzheimer del perro", por sus similitudes clínicas y fisiopatológicas con esta enfermedad de los humanos (6), es un síndrome demencial, neurodegenerativo, de carácter progresivo, que tienden a padecer algunos caninos a lo largo de su vida geriátrica (4,7-9). Esta patología se presenta generalmente en perros mayores de 7 años (6), algunos de los cuales exhiben contundentes signos clínicos relacionados a la disminución de la capacidad cognitiva.

El término cognición hace referencia a procesos mentales como percepción, conciencia, aprendizaje, memoria y toma de decisiones, que permiten al individuo obtener información del medio ambiente para así, tomar decisiones, actuar y desenvolverse normalmente (10). En este 
sentido, el déficit cognitivo observado en pacientes con SDC se refiere a la disminución en la capacidad de recopilar información, procesarla, retenerla y tomar decisiones $(3,8,11-13)$, dando origen a cambios comportamentales que al ser considerados por algunos propietarios, como signos "normales" del envejecimiento, no son reportados al médico veterinario. Estos cambios incluyen la falta de reconocimiento hacia los miembros de la familia, dificultad para realizar tareas simples como comer, hacer deporte y alteraciones en el ciclo sueño-vigilia (14), provocando limitación del desenvolvimiento social y sentido de supervivencia en el animal afectado, lo que finalmente puede conducir al rechazo de ciertos propietarios para asumir el cuidado de este tipo de pacientes, aumentando el riesgo de algunas mascotas a ser sacrificadas.

Por otro lado, el sacrificio del animal también se refleja en una disminución en la calidad de vida de su familia, ya que se genera en ella sentimientos de dolor y pérdida.

Tras considerar las implicaciones médicas y sociales de este tipo de padecimientos y teniendo en cuenta la importancia de hacer un reconocimiento preciso de esta patología, frente a signos de decline cognitivo leve provocados por el envejecimiento normal, el presente artículo se propone describir de manera detallada las características clínicas y fisiopatológicas propias de este síndrome demencial canino y de igual manera presenta las herramientas diagnósticas y terapéuticas que buscan detener la progresión de los signos clínicos de esta enfermedad, la cual es considerada hoy como uno de los trastornos neurodegenerativos de mayor impacto, asociados a la edad en la población canina geriátrica.

Presentación clínica del SDC. Los signos que incluyen alteraciones comportamentales en perros geriátricos, algunas veces son considerados como rasgos propios del proceso de envejecimiento, sin embargo, es importante diferenciar entre aquellas alteraciones comportamentales que están relacionadas con un daño serio de los procesos cognitivos, es decir, "envejecimiento patológico" y una disminución leve de la actividad psicomotora o "envejecimiento normal" $(2,13,15-16)$.

Los cambios comportamentales son característicos de cada paciente afectado, pudiendo observarse distintos grados de alteración cognitiva, por ejemplo, algunos perros pueden llegar a desconocer a los miembros de su familia y otros, con un grado menor de déficit cognitivo, pueden recordar sin dificultad las instrucciones aprendidas durante el entrenamiento casero previo (14).

Probablemente, la alteración de los hábitos de micción, pero no de defecación, es el más frecuente de los signos observados por los amos en los pacientes con SDC (14); así, la poliuria puede ocurrir sin la presencia de enfermedades secundarias o sin que ocurran cambios medioambientales como la falta de acceso a un área apropiada de evacuación y la presencia o no de los propietarios. Adicionalmente, en algunas ocasiones se reportan casos en los que el perro afectado presenta episodios de confusión y desorientación (17), en los que la mascota puede perderse dentro de la casa o el jardín, atascarse en una esquina, ir a la puerta equivocada o al lado equivocado de la puerta. Los signos clínicos también incluyen menor interacción con los propietarios, lentitud para obedecer órdenes, alteraciones en el ciclo sueñovigilia, vocalización excesiva, intolerancia al ejercicio, dificultad para subir escaleras, aumento de la irritabilidad, nuevos miedos o fobias y en algunos casos aislados, los perros pueden apegarse más a sus propietarios y seguirlos a todos lados. $(14,17)$.

Dada la amplia variedad de alteraciones comportamentales reportadas, algunos autores han sugerido el uso de escalas de clasificación para las mismas, como la propuesta por Landsberg (3), donde los signos clínicos pueden ser categorizados bajo los siguientes tópicos: 1. Desorientación espacial y/o confusión, 2. Alteración en las capacidades de aprendizaje y memoria (pérdida de hábitos de aseo en casa, pérdida de la capacidad para obedecer algunas ordenes o tareas previamente aprendidas), 
3. Actividad repetitiva o disminución de esta, 4. Alteración y disminución de las relaciones sociales, 5. Disminución de la percepción y/o de la capacidad de respuesta, 6. Aumento de la ansiedad o inquietud, 7. Alteración del apetito, asociada a estados de confusión que les impide ubicar su alimento, 8. Alteración de los ciclos diurno-nocturno (sueño-vigilia). Aunque la alteración del ciclo sueño vigilia característica de algunos perros con SDC ha sido más difícil de interpretar, los resultados obtenidos a partir de estudios realizados en humanos con enfermedad de Alzheimer (EA) sugieren que el disturbio de este proceso puede ser el resultado de una alteración en el ritmo circadiano en perros que padecen $\operatorname{SDC}(18,19)$.

Prevalencia. Considerando los reportes que sugieren la presencia a nivel mundial de más de 52 millones de caninos alrededor de los 7 años edad (20), y teniendo en cuenta que debido a su condición geriátrica, esta es la población canina con mayor riesgo de padecer $\operatorname{SDC}(6,19)$, numerosos estudios de impacto regional, han diseñado e implementado diferentes cuestionarios observacionales, a través de los cuales, los propietarios de mascotas caninas geriátricas son entrevistados para detectar posibles cambios comportamentales y, con esto, lograr determinar la prevalencia de SDC en los animales evaluados. En este sentido, un estudio realizado en Italia que incluyó 124 perros geriátricos, reveló una prevalencia de SDC cercana al $50 \%$, en la que 75 perros mayores de 7 años, presentaron signos coincidentes con la enfermedad (13). De igual manera, otro estudio (1) realizado con 180 perros entre los 11 y 16 años de edad, encontró que el $28 \%$ de los perros entre 11 y 12 años, mostraron algún grado de déficit cognitivo, mientras que aquellos individuos entre 15 y 16 años tenían una probabilidad cercana al $68 \%$ de sufrir la enfermedad, lo cual sugiere una estrecha relación entre el proceso de envejecimiento y la probabilidad de padecer SDC.

No obstante, los datos sobre la prevalencia mundial de este síndrome demencial aún no han sido obtenidos, en parte, debido a la tendencia de un gran número de propietarios a no reportar al médico veterinario los posibles cambios comportamentales en sus mascotas geriátricas (13), lo que probablemente ha limitado la obtención de datos precisos que estimen la prevalencia del SDC a nivel mundial.

Bases fisiopatológicas del SDC. EI término neurodegeneración define la muerte neural patológica observada en algunas enfermedades neurodegenerativas como el SDC. Esta enfermedad se caracteriza morfológicamente por una disminución en el número de neuronas colinérgicas en la corteza cerebral y el hipocampo (áreas especialmente relacionadas con cambios en el comportamiento cognitivo y la memoria) $(3,21)$. Aunque el origen de esta disminución es desconocido, algunos autores han sugerido al estrés oxidativo y la acumulación de péptido beta-amilode $(\beta A)$, como posibles factores causales de los signos clínicos observados en SDC (2).

El péptido $\beta A$ juega un papel importante en la fisiopatología de la demencia canina $(2,8,22-29)$ y de la EA en humanos (2) ya que su acumulación genera efectos neurotóxicos (6) como degeneración de las sinapsis y pérdida de neuronas colinérgicas; adicionalmente, su presencia y grado de acumulación han sido relacionados con la severidad de los signos clínicos $(1,6,8,22,30-31)$.

Por su parte, el estrés oxidativo provoca lesiones a nivel de proteínas, lípidos y ácidos nucleicos de diversas neuronas $(2,8,32-$ 33). De acuerdo con algunos autores, este daño oxidativo es un mecanismo fundamental para el desarrollo de las enfermedades que cursan con disfunción cognitiva asociada a la edad (34), ya que el paso del tiempo en el cerebro provoca agregación de sustancias oxidantes y disfunción de los mecanismos protectores como la superóxido dismutasa y la vitamina E (35), lo que puede potencialmente dañar la función neuronal y provocar su muerte (2). Esta muerte neuronal conduce a la depleción de neurotransmisores de tipo excitatorio como la acetil-colina que interviene en prácticamente todas las funciones cognitivas y especialmente en la memoria; la dopamina que se relaciona con 
el control del movimiento (motricidad), las emociones y la capacidad de sentir placer; la noradrenalina asociada con la vigilia, la atención y la capacidad de asimilar nuevos datos; y la serotonina que se relaciona con el estado anímico y el control del sueño $(6,21,23,36)$. En este sentido, tales alteraciones neuroquímicas, acompañan la manifestación de varios de los signos clínicos observados en pacientes con SDC.

Diagnóstico. En la práctica veterinaria, los problemas conductuales son comunes y además, suelen ser de origen multifactorial $(8,9,31)$. Así, considerando la variedad e inespecificidad de los signos clínicos asociados al SDC, es importante utilizar la anamnesis como herramienta para obtener datos específicos del paciente y asegurarse que el propietario ha proporcionado una lista completa de todos los signos comportamentales y médicos de su mascota, ya que tal información podría proporcionar un sustento sólido en el hallazgo de posibles problemas médicos que puedan ser responsables de la presentación o exacerbación de los signos clínicos (3), además, el examen clínico y la evaluación neurológica mediante el uso de pruebas de evaluación cognitiva, podrían conducir al médico veterinario a la obtención de un diagnóstico temprano; no obstante, el único diagnóstico confiable es el que se basa en estudios anatomopatológicos post mortem de muestras encefálicas, de tal forma que se pueda confirmar microscópicamente la presencia de los cúmulos de $\beta A$ (6).

De esta manera, la escasez de pruebas diagnósticas de alta confiabilidad que garanticen la presencia o no de la enfermedad, otorga a la identificación temprana de los signos clínicos, un papel crucial para el establecimiento de un buen pronóstico en términos del mejoramiento y extensión en la calidad de vida de los pacientes afectados.

En este sentido y de acuerdo con algunos autores, la forma más efectiva de detectar esta condición es a través de la instauración de cuestionarios comportamentales en la clínica geriátrica rutinaria $(2,8)$, obtenidos a partir de diversas investigaciones $(15,34)$ que han permitido el desarrollo de una serie de cuestionarios que pretenden clasificar el comportamiento de los pacientes afectados. Dentro de estos cuestionarios de evaluación cognitiva, se encuentran las pruebas neurofisiológicas sistemáticas que buscan clasificar el daño cognitivo a través de métodos como el Aparato Modificado de Prueba General de Winsconsin (Universidad de Toronto), en el que los perros son recompensados por cada respuesta acertada que obtengan. En términos generales, en esta prueba los perros tienen acceso a una bandeja extraíble que contiene tres alimentos empotrados en pozos, los cuales pueden ser cubiertos para poner a prueba el aprendizaje visual y la memoria (37-38).

Por ejemplo, una prueba de discriminación consiste en presentar a los perros una opción de respuesta frente a tres objetos, donde uno es diferente y los otros dos son idénticos. Para realizar de forma adecuada esta prueba, se requiere que el sujeto aprenda que el objeto extraño está asociado con un premio de comida y que recuerde esta regla general al ser evaluado en una ocasión posterior. Además, para distribuir el sentido del olfato para la solución, se coloca igual cantidad de comida en una posición inaccesible de los objetos que no ofrecen recompensa (2).

Estas pruebas neurofisiológicas permiten la evaluación objetiva y cuantitativa de los déficits en el aprendizaje y la memoria, sin depender de los cuestionarios realizados a los propietarios y esta evaluación es posible gracias a la implementación de tres objetivos específicos: 1. Identificación de los cambios cognitivos no subjetivos, que sean característicos del envejecimiento en los perros, 2. Caracterización de las bases neurobiológicas de la disminución en la capacidad cognitiva, debido al envejecimiento, y 3. Elaboración de potenciales intervenciones con el objeto de suprimir o minimizar los efectos sobre la calidad de vida del animal (2,37-38).

Igualmente y teniendo en cuenta que las dos capacidades cerebrales susceptibles a declinar frente al paso de los años, memoria espacial (la habilidad para recordar la 
localización de la comida, por ejemplo) y memoria de reconocimiento de objetos (la habilidad para recordar objetos vistos con 10-120 segundos de anticipación) (8), son afectadas en los procesos neurodegenerativos, varios estudios han desarrollado ciertas escalas y criterios de evaluación indirecta de demencia en perros (39). Por ejemplo, ha sido propuesto un índice de evaluación de demencia que discrimina entre normal, predemencia y demencia (17). Adicionalmente (40), presentó la escala A.R.C.A.D (evaluación de desordenes cognitivos y afectivos relacionados con la edad), donde el comportamiento del perro es evaluado indirectamente por medio de un cuestionario formal realizado al propietario, con el fin de evaluar el déficit mediante una escala de evaluación de 1 a 5 .

No obstante, para la detección del grado de disfunción cognitiva, habrá de tenerse en cuenta el tipo de prueba realizada y el aumento en la variabilidad de las habilidades cognitivas de los perros afectados $(8,13)$. De acuerdo con (2), la terminología actual de clasificación basada en los déficits cognitivos y los cambios comportamentales observados en perros geriátricos, es probable que necesite de un mayor perfeccionamiento o revisión por parte de los investigadores, ya que aún no es claro si la clasificación del comportamiento en perros examinados, presenta un vínculo directo con las medidas clínicas y de laboratorio observadas en pacientes afectados con SDC; además, se desconoce actualmente si los resultados del laboratorio se pueden traducir directamente en los resultados obtenidos a partir de la práctica clínica. Es importante hacer esta distinción, debido a que aún no se encuentra establecido si las pruebas de aprendizaje, de memoria y de procesamiento cognitivo realizadas junto con las pruebas de laboratorio específicas, involucran la participación de los mismos circuitos cerebrales que están comprometidos en SDC. Debido a esto, el clasificar un perro con SDC en base a las puntuaciones neuropsicológicas resulta bastante complejo, ya que aquellos estudios que han incluido pruebas neuropsicológicas y cuestionarios para evaluar los cambios comportamentales, solo se han realizado en un mismo grupo de perros (2).
Adicionalmente, son varios los dominios cognitivos que se ven afectados durante el SDC (lenguaje, memoria, habilidades visoespaciales); y la observación de un signo, no es suficiente para la instauración de un tratamiento. No obstante, la aparición de un deterioro cognitivo se puede llegar a traducir en el empeoramiento de los signos existentes, y pone de manifiesto la importancia de hacer seguimiento a las evaluaciones clínicas (8).

En conclusión, para determinar si un perro presenta signos de disfunción cognitiva, el médico veterinario debe confiar en la información otorgada por el propietario en la historia clínica; no obstante, con el diseño y aplicación de un cuestionario cuidadosamente elaborado, es probable que los signos de SDC, sean detectados durante las primeras etapas de desarrollo, y en especial si se trata de animales que hayan tenido previamente un alto nivel de entrenamiento (3).

\begin{abstract}
Alternativas terapéuticas para el tratamiento del SDC. Actualmente se dispone de varias estrategias terapéuticas que han sido desarrolladas a lo largo de diferentes estudios realizados con caninos geriátricos afectados por SDC.
\end{abstract}

De tipo comportamental. Una consecuencia de las patologías asociadas a la edad en perros, es la pérdida de los recuerdos adquiridos durante el entrenamiento casero o profesional y debido a este efecto, el perro puede perder su habilidad para realizar tareas simples o para responder comandos previamente aprendidos. Frente a este tipo de cambios comportamentales que pueden ser observados en pacientes con SDC, la instauración de una terapia comportamental y reentrenamiento del animal en estados tempranos de la enfermedad, han sido sugeridas como herramientas terapéutica adecuadas y adicionales al tratamiento farmacológico. Reeducar a perros con disfunción cognitiva, requiere paciencia y el uso de órdenes muy simples con una clara recompensa y es importante que el propietario inicie este reentrenamiento lo antes posible, para evitar el establecimiento de comportamientos indeseados en la mascota $(2,6)$. 
Farmacológico. Parte del tratamiento farmacológico tiene como objetivo restablecer los niveles de neurotransmisores para evitar que el proceso degenerativo avance demasiado rápido. La mayor parte de tratamientos utilizados para personas afectadas con la EA, aún no han sido probados en perros, así que en la práctica clínica se hace necesario utilizar el criterio científico y profesional para ver cuales tratamientos funcionan y cuáles no. No obstante, existen algunas opciones disponibles como la selegilina, primer agente terapéutico aprobado por la FDA en 1998 para uso en perros $(5,41)$; este es un inhibidor selectivo e irreversible de la enzima monoamino-oxidasa B (MAO B), que aumenta los niveles de dopamina y además posee efecto antioxidante, disminuye la muerte celular y favorece la síntesis de factores de crecimiento neuronal. En cerebros caninos, la selegilina incrementa la 2-feniletilamina, la cual funciona como neuromodulador y potencializa las funciones de la dopamina y las catecolaminas (3). Un doble estudio utilizando selegilina, demostró la disminución en la progresión de los cambios degenerativos en pacientes con EA y un mejoramiento significativo en perros con SDC (1). Por lo anterior, la regulación en la concentración de neurotrasmisores ha mostrado ser la terapia farmacológica con mayor efectividad frente a la progresión de signos clínicos de perros con SDC.

Por otro lado, existen otras opciones farmacológicas para el tratamiento del SDC en caninos geriátricos, tales opciones incluyen fármacos que favorecen la circulación vascular cerebral, como la nicergolina y la propentofilina; y fármacos que potencien el sistema noradrenérgico como el adrafinil y el modafinil (42).

Nutricional. Tras reconocer el papel de ROS (especies reactivas al oxígeno) en las enfermedades neurodegenerativas, algunos investigadores han recomendado, por una parte, intentar reducir la cantidad de radicales libres formados a partir de influencias exógenas ambientales y, por otra parte, la inclusión en la dieta de suplementos nutricionales, los cuales han demostrado poseer un papel potencialmente valioso a la hora de maximizar los beneficios de la terapia psicofarmacológica, en términos de aumento de la calidad de vida al observar cambios positivos significativos en el comportamiento de caninos que sufren $\operatorname{SDC}(2,5,33)$.

Dentro de los efectos benéficos atribuidos a los productos antioxidantes, se encuentra la potencialización de la función mitocondrial durante la vejez, lo que resulta en disminución de la producción de ROS e incremento funcional de los sistemas antioxidantes $(2-3,8,34)$. Como resultado de estos efectos, algunos autores han sugerido un mejoramiento en la capacidad cognitiva de pacientes afectados con SDC (43) y adicionalmente, una variedad de estudios han mostrado que la ingesta de frutas y vegetales, puede disminuir el riesgo de sufrir el decline cognitivo asociado a la edad en roedores, perros, e inclusive en humanos, atribuyendo esta propiedad a sus capacidades antioxidantes y antiinflamatorias $(3,5,34)$.

Tales investigaciones, han permitido la identificación de una cantidad de compuestos antioxidantes como las vitaminas $\mathrm{E}$ y $\mathrm{C}$, el beta-caroteno, el selenio, la L-carnitina y el ácido alfa lipóico, cuya asociación se ha comprobado que mejora la función mitocondrial en neuronas de animales viejos, haciendo que funcionen a niveles comparables a los de animales jóvenes, además, tienen un efecto sinérgico en el mejoramiento de la memoria. Igualmente, ciertos autores sugieren que el Gingko Biloba, vegetal que además de poseer efecto antioxidante, presenta una variedad de propiedades tales como antiinflamatoria, vasodilatadora cerebral, potenciadora de la función mitocondrial e inhibidora de la enzima MAO $(3,13,44)$, genera una disminución de la severidad de los signos clínicos observados en pacientes con SDC. Adicionalmente, compuestos naturales de origen animal como el propóleo, del cual nuestro grupo de investigación estudia sus propiedades neuroprotectoras, actualmente son valorados por sus cualidades antioxidantes y neuroprotectoras (45); no obstante, aún no existen estudios que describan efectos positivos de este compuesto sobre SDC. 
Otras investigaciones incluyen el estudio de la función de ciertas moléculas clasificadas como cofactores mitocondriales (acido lipoico, I-carnitina), los cuales pueden potenciar la función de la mitocondria de células viejas, provocando una menor producción de ROS durante la respiración aerobia. La suplementación alimenticia con estos cofactores mitocondriales, incrementa sus concentraciones dentro de las células y restaura la unión a enzimas específicas, lo que restituye la eficiencia mitocondrial y reduce el daño oxidativo al RNA (46). Igualmente, se ha sugerido el uso de cofactores mitocondriales junto con antioxidantes, con el objeto de provocar un mejoramiento en los procesos de aprendizaje y memoria, por medio de una acción sinérgica (18).

Por otro lado, en el sector comercial ya se encuentran disponibles productos como la dieta terapéutica de Hill's, conocida como Prescription dietR Canine $b / d R$, (Hills Pet Nutrition, Topeka, KS), disponible para el tratamiento de la disfunción cognitiva en perros senior. La eficacia de esta dieta fue evaluada utilizando pruebas neurofisiológicas durante más de dos años. El objetivo del estudio fue suplementar la dieta de perros, con cofactores mitocondriales y antioxidantes de amplio espectro, con el fin de mejorar las defensas antioxidantes y disminuir la acumulación de ROS y sus efectos tóxicos; después de una prolongada evaluación, los resultados indicaron que estos productos pueden ayudar retrasar el decline cognitivo relacionado con la edad (3). No obstante, para desarrollar una dieta suplementada con antioxidantes, se debe tener en cuenta que la selección de los componentes, los rangos de dosis y la vía de administración, varían considerablemente entre especies. Algunos antioxidantes son absorbidos más rápidamente que otros, por factores propios de cada especie, debido a las diferencias metabólicas intrínsecas de biodisponibilidad; así, diferentes especies pueden beneficiarse de diferentes tipos de antioxidantes, pero no todas las especies pueden beneficiarse de los mismos antioxidantes (2).

En conclusión, esta práctica requiere de mayor investigación para poder integrar la suplementación alimenticia como un componente terapéutico en los casos de SDC, lo que a su vez, podría llegar a ser soportado por algunos autores que han sugerido una variedad de efectos benéficos en casos de EA, a partir de la implementación de dietas ricas en sustancias antioxidantes $(2-3,8,34)$.

Hábitos de vida más saludables. Para perros geriátricos afectados con SDC el ejercicio físico, una correcta alimentación y la suplementación alimenticia con sustancias antioxidantes, se constituyen como herramientas útiles para retrasar tanto la aparición del proceso, como la velocidad de evolución del mismo. La actividad física mejora el flujo sanguíneo al cerebro y así puede reducir el riesgo de infarto, demencia y declive cognitivo. La actividad podría estimular el crecimiento de las células nerviosas en el hipocampo, la región del cerebro que participa en funciones de la memoria, y de acuerdo con varios estudios, ello ayudaría al cerebro a construir una especie de reserva para prevenir un futuro deterioro mental $(3,6)$.

En conclusión, dado el papel del médico veterinario como velador de la calidad de vida de los caninos geriátricos, durante la práctica clínica resulta de gran importancia el entrenamiento en el conocimiento, detección de signos clínicos asociados al Síndrome de Disfunción Cognitiva y además, el criterio científico con el que sea abordado el manejo terapéutico para esta enfermedad, haciendo énfasis en el diagnóstico de la condición para instaurar algunos de las alternativas terapéuticas disponibles.

Por otra parte, teniendo en cuenta la relación existente entre este síndrome demencial y el proceso de envejecimiento, los perros en riesgo de padecer la enfermedad son muchos y más si se considera el continuo crecimiento de la población canina geriátrica, por lo tanto, se hacen necesarias futuras investigaciones en el conocimiento, control y prevención de esta patología, para disminuir el impacto generado por sus efectos. 


\section{REFERENCIAS}

1. Neilson J, Hart B, Cliff K, Ruehl W. Prevalence of behavioral changes associated with age-related cognitive impairment in dogs. JAVMA 2001; 218(11):1787-1791.

2. Head E, Zicker S. Nutraceuticals, aging, and cognitive dysfunction. Vet Clin North Am Small Anim Pract 2004; (34):217-228.

3. Landsberg G. Therapeutic agents for the treatment of cognitive dysfunction syndrome in senior dogs. Prog Neuropsychopharmacol Biol Psychiatry 2005; 29(3):471-479.

4. Ruehl WW, Bruyette DS, DePaoli A, Cotman CW, Head E, Milgram NW, Cummings BJ. Canine cognitive dysfunction as a model for human age-related cognitive decline, dementia and Alzheimer's disease: clinical presentation, cognitive testing, pathology and response to I-deprenyl therapy. Prog Brain Res 1995; (106):217-225.

5. Zicker S. Cognitive and behavioral assessment in dogs and pet food market applications. Prog Neuropsycho Biol Psychiatry 2005; (29): 455-459.

6. Pérez-guisado J. El Síndrome de disfunción cognitiva en el perro. Disponible en. RECVET [en línea] 2007 [fecha de acceso 05 de junio de 2009]; 2(01-04). URL disponible en: http:// www.veterinaria.org/revistas/recvet/ n01a0407/01a040701.pdf.

7. Borras D, Ferrer I, Pumarola M. Agerelated Changes in the Brain of the Dog. Vet Pathol 1999; 36(3):202-211.

8. Head E. Brian aging in dogs: Parallels with human brain aging and Alzheimer's disease. Vet Ther 2001; 2(3):247-260.
9. Koppang N. Canine ceroid lipofuscinososis-a model for human neuronal ceroid lipofuscinososis and aging. Mech Ageing Dev 1973; (2):421-445.

10. Shettleworth SJ. Animal cognition and animal behaviour. Anim Behav 2001; 61(2):277-286.

11. Boutet I, Ryan M, Kulaga V, McShane C, Christie LA, Freedman M, Milgram NW. Age-associated cognitive deficits in humans and dogs: a comparative neuropsychological approach. Prog Neuropsychopharmacol Biol Psychiatry $2005 ; 29(3): 433-441$.

12. Wang $X$, Ding H. Alzheimer's disease: epidemiology, genetics, and beyond. Neurosci Bull 2008; 24(2):105-109.

13. Osella M, Re G, Odore R, Girardi C, Badino $P$, Barbero $R$, et.al. Canine cognitive dysfunction syndrome: Prevalence, clinical signs and treatment with a neuroprotective Natraceutical. Appl Anim Behav Sci 2007; (105):297-310.

14. Frank D. Cognitive dysfunction in dog. In: Hill's European Symposia on Canine Brain Ageing [en línea] 2002. [fecha de acceso 27 de mayo de 2009]. URL disponible en: http://www.ivis. org/proceedings/Hills/brain/frank. $\operatorname{pdf}$ ? $L A=1$.

15. Rofina JE, van der Meer I, Goossens M, Secrève M, Ederen van AM, Schilder $M$, et.al. Preliminary inquiry to assess behavior changes in aging pet dogs. En: Bely M, Apathy A editores. Amyloid and Amyloidosis IX; Budapest, Hungary: Apathy A editores; 2001.

16. Peinado MA, del Moral ML, Esteban FJ, Martínez Lara $E$, Siles $E$, Jiménez $A$, et.al. Envejecimiento y neurodegeneración: bases moleculares y celulares. Rev Neurol 2000; 31(11):1054-1065. 
17. Landsberg G, Ruehl W. Geriatric behavioral problems. Vet Clin North Am Small Anim Pract 1997; 27(6):1537-1559.

18. Cummings BJ, Su JH, Cotman CW, White $\mathrm{R}$, Russell M. $\beta$-Amyloid accumulation in aged canine brain. A model of early plaque formation in Alzheimer's disease. Neurobiol Aging 1993; (14):547-556.

19. Gerosa R. Geriatría canina: trastornos y lesiones orgánicas en perros de edad avanzada. Buenos Aires: Ed Interamerica; 2007.

20. Association AVM. US Pet Ownership \& Demographics Sourcebook. Schaumburg, Illinois: American Veterinary Medical Association; 1997.

21. Pérez J. La descripción de los ovillos neurofibrilares en la enfermedad de Alzheimer. Rev Esp Patol 2007; (40) 1:60-65.

22. Cummings $B J$, Satou $T$, Head E, Milgram NW, Cole GM, Savage MJ, et.al. Diffuse plaques contain $C$-terminal $A \beta 42$ and not $A \beta 40$ : evidence from cats and dogs. Neurobiol Aging 1996; 17(4):653-659.

23. Pugliese $M$, Gangitano $C$, Ceccariglia $\mathrm{S}$, Carrasco J, Rodríguez M, Michetti F, et.al. Canine cognitive dysfunction and the cerebellum: Acetylcholinesterase reduction, neuronal and glial changes. Brain Res Rev 2007; (1139):85-94.

24. Walker LC: Animal models of cerebral beta-amyloid angiopathy. Brain Res Rev 1997; (25): 70-84.

25. Galdzicki Z, Fukuyama R, Wadhwani K, Rapoport S, Ehrenstein G. $\beta$-Amyloid increases choline conductance of PC12 cells: Possible mechanism of toxicity in Alzheimer's disease. Brain Res Rev 1994; (646): 332-336.

26. Kiatipattanasakul W, Nakamura $S$, Hossain M, Nakayama $H$, Uchino T, Shumiya $S$, et al. Apoptosis in the aged dog brain. Acta Neuropathol 1996; (92):242-248.
27. Su JH, Anderson AJ, Cummings BJ, Cotman CW. Immunohistochemical evidence for apoptosis in Alzheimer's disease. Neuro Report 1994; (5):2529-2533.

28. Rofina JE, Singh K, SkoumalovaVesela $A$, van Ederen AM, van Asten AJ, Wilhelm J, et al. Histochemical accumulation of oxidative damage products is associated with Alzheimerlike pathology in the canine. Amyloid 2004; 11(2):90-100.

29. Dimakopoulos A, Mayer R. Aspects of Neurodegeneration in the Canine Brain. J Nutr 2002; 132(Supl 1):1579-1582.

30. Head $E$, Callahan $H$, Muggenburg BA, Cotman CW, Milgram NW. Visualdiscriminación learning ability and beta-amyloid accumulation in the dog. Neurobiol Aging 1998; 19(5):415-425.

31. Colle MA, Hauw JJ, Crespeau F, Uchiara $\mathrm{T}$, Akiyama H, Checler $\mathrm{F}$, et al. Vascular and parenchymal $A b$ deposition in the aging dog: correlation with behavior. Neurobiol Aging 2000; (21):695-704.

32. Ames BN, Shigenaga MK, Hagen TM. Oxidants, antioxidants, and the degenerative diseases of aging. Proc Natl Acad Sci USA 1993; (90): 7915-7922.

33. Head E. Oxidative Damage and Cognitive Dysfunction: Antioxidant Treatments to Promote Healthy Brain Aging. Neurochem Res 2009; (34): 670-678.

34. Cotman C, Head E, Muggenburg B, Zicker $\mathrm{S}$, Milgram N. Brain aging in the canine: a diet enriched in antioxidants reduces cognitive dysfunction. Neurobiol Aging 2002; (23):809-818.

35. Kiatipattanasakul W, Nakamura S, Kuroki K, Nakayama H, Doi K. Immunohistochemical detection of anti-oxidative stress enzymes in the dog brain. Neuropathology 1997; (17):307-12. 
36. Lieberman D, Mody I, Pike C, Cotman C. â-Amyloid (25-35) prolongs opening of NMDA channels through an intracellular pathway. Soc Neurosci 1994; (20):602.

37. Adams B, Chan A, Callahan H, Siwak C, Tapp D, Ikeda-Douglas C, et al. Spatial learning and memory in the dog as a model of cognitive aging. Behav Brain Res 2000; 108(1):47-56.

38. Milgram NW, Head E, Weiner E, Thomas E. Cognitive functions and aging in the dog: acquisition of nonspatial visual tasks. Behav Neurosci 1994; (108):57-68.

39. Uchino T, Kida M, Baba A, Ishii K, Okawa N, Hayashi Y, Shumiya S. Senile dementia in aged dogs and the diagnostic standards. Proceedings of the 17th Symposium of Japan Society for Biomedical Gerontology. Biomed Gerontol 1995; (19):24-31.

40. Pageat $P$. Description, clinical and histological validation of the A.R.C.A.D. score (evaluation of age-related cognitive and affective disorders). En: Lynne Seibert DVM, MS editors. Newsletter of the American Veterinary Society of Animal Behavior. Third International Congress on Behavioural Medicine; Boston 7-8 de Agosto de 2001. Boston: Lynne Seibert DVM, MS editors; 2001.

41. Campbell S, Trettien A, Kozan B. A non comparative open-label study evaluating the effect of selegiline hydrocloride in a clinical setting. Vet Ther 2001; (2):24-39.
42. Landsberg G. Decline in Senior Pets Therapeutic Options for Cognitive. J Am Anim Hosp Assoc 2006; 42: 407-413.

43. Head E, Liu J, Hagen T, Muggenburg B, Milgram N, Ames B, Cotman C. Oxidative damage increases with age in a canine model of human brain aging. J Neurochem 2002; (82): 375-381.

44. Launer LJ, Andersen K, Dewey M, Lettenneur $L$, Ott A, Amaducci $L$. Rates and risk factors for dementia and Alzheimer's disease. Results from EURODEM pooled analyses. Neurology 1999; (52):78-74.

45. Shimazawa M, Chikamatsu S, Morimoto $\mathrm{N}$, Mishima S, Nagai $\mathrm{H}$, Hara $\mathrm{H}$. Neuroprotection by Brazilian Green Propolis against In vitro and In vivo Ischemic Neuronal Damage. Ecam 2005; 2(2):201-207.

46. Liu J, Killilea DW, Ames BN. Ageassociated mitochondrial oxidative decay: improvement of carnitine acetyltransferase substrate-binding affinity and activity in brain by feeding old rats acetyl-L-carnitine and/or R-alpha -lipoic acid. Proc Natl Acad Sci USA 2002; 99(4):1876-1881. 